\title{
Editorial introduction to the special issue on 'Strategic queueing: game-theoretic models in queueing theory'-part 1
}

\author{
Antonis Economou ${ }^{1} \cdot$ Vidyadhar Kulkarni $^{2}$
}

Accepted: 21 November 2020 / Published online: 18 December 2020

(c) The Author(s), under exclusive licence to Springer Science+Business Media, LLC part of Springer Nature 2020

We are very pleased to guest edit this special issue of Queueing Systems, on Strategic Queueing. This subfield of Queueing Theory studies situations where multiple queueing entities, like customers, servers, and/or administrators, are active decision makers. This is in contrast to classical Queueing Theory that considers them as passive entities that do not make decisions, or at least that their behavior is exogenously given. The conflict of interests among the various agents of a queueing system requires the use of concepts and methodological tools from Game Theory. Thus, Strategic Queueing can be defined as the study of queueing systems from a game-theoretical viewpoint.

More concretely, an economic evaluation of a queueing system cannot be credible, unless the strategic nature of its various agents is taken into account. This point of view was first assumed about 50 years ago in a seminal paper by Naor [9], who studied the join-or-balk dilemma for the customers in the $\mathrm{M} / \mathrm{M} / 1$ queue, when its queue length is observable. He also considered the problem of a social planner and a monopolist who optimize the social welfare and the revenue, respectively, considering customer strategic behavior. Edelson and Hildebrand [2] complemented Naor's study by considering the same problems for the unobservable version of the system. In their model, the arriving customers are not allowed to observe the number of customers in the system and make their join-or-balk decisions relying solely on the knowledge of its operational and economic parameters, assuming that the system has reached stochastic steady state. Since then, the literature on strategic behavior in queueing systems has grown considerably. It is fair to say that it is by now a mature subfield of Queueing

Antonis Economou

aeconom@math.uoa.gr

Vidyadhar Kulkarni

vkulkarn@email.unc.edu

1 Department of Mathematics, Section of Statistics and Operations Research, National and Kapodistrian University of Athens, 15784 Panepistemiopolis, Athens, Greece

2 Department of Statistics and Operations Research, University of North Carolina, Chapel Hill, NC 27599, USA 
Theory that complements the studies of the performance evaluation, optimal design and optimal control of service systems.

The first book on Strategic Queueing was written by Hassin and Haviv [4], who provided a nice overview of the basic methodology and the early results in this field. This book had an enormous impact in popularizing the viewpoint of Strategic Queueing and in some sense shaped the field. The monographs by Stidham [10] and Hassin [5] contained further material and overviews about models and methodologies in this subfield of Queueing. In addition, several overviews on important issues of Strategic Queueing have been recently published. Ibrahim [8] and Economou [1] summarized the impact of information structures on strategic behavior in queueing systems. Ghosh and Hassin [3] provided a detailed literature review about the issue of inefficiency in queueing systems. A related topic is the applications of coordination mechanisms in the framework of queueing systems. Haviv and $\mathrm{Oz}[6,7]$ summarized the various coordination mechanisms that have been proposed in the literature, classified them and proposed new ones.

Queueing Systems, recognizing the importance of Strategic Queueing within Queueing Theory, devoted a special issue to celebrate the $50^{\text {th }}$ anniversary of the publication of the first strategic queueing paper. The first part of this special issue comprises five papers:

Nimrod Dvir, Refael Hassin and Uri Yechiali consider an unobservable two-station tandem queueing system, attended by an alternating server, i.e. a polling system, and study the strategic customer behavior under two threshold-based operating policies that are applied by the profit maximizing server. They show that the first policy leads to a mixture of Follow-the-Crowd and Avoid-the-Crowd behavior, whereas the second policy leads only to an Avoid-the-Crowd behavior. Moreover, they study analytically and numerically the optimal performance of the system, when customers act according to their equilibrium strategies.

N. Hemachandra, Kishor Patil and Sandhya Tripathi study the interaction between arriving customers and a service station, when the arrival rates depend on the Qualityof-Service (QoS) that the customers experience. The interaction is studied under two different QoS measures and the authors investigate the behavior of equilibrium points and equilibrium sets associated with this interaction. In particular, sufficient conditions for the existence of equilibrium sets for the $M / M / 1$ queue are provided, and then the analysis proceeds to the more general case of GI/M/1 queues.

Yoav Kerner and Ophir Shmuel-Bittner study customer strategic behavior in a hybrid M/M/1 queue with retrials. This system comprises an ordinary waiting queue and an orbit. Arriving customers enter the ordinary queue or the orbit according to a predefined threshold policy. Balking and reneging are prohibited, and the decision of the customers lies in choosing their retrial rate. The authors study the equilibrium and socially optimal retrial rates, under a cost structure that considers waiting and retrial costs. Finally, they quantify the discrepancy between individual and social optimality using the Price-of-Anarchy measure.

Tesnim Naceur and Yezekael Hayel study the impact of queue length information on the behavior of customers and on the associated social welfare. They consider policies, parameterized by a nonnegative integer $D$, where the administrator of a system informs the arriving customers about the exact queue length when it is less than or equal to $D$, 
otherwise he conceals it. They show that, in some circumstances (and particularly when the queue is overloaded), providing information up to a particular level of occupancy yields a better expected social welfare than always providing queue length information to new customers.

S. Srivatsa Srinivas and Rahul R. Marathe study a single server Markovian queue with a finite buffer and two types of customers: patient customers who always join and impatient customers who balk if the queue is too long. The service rate is either fast or slow, and the impatient customers evaluate their expected utility after observing the queue. The authors show that equilibrium with threshold strategies always exists and that there may be multiple equilibria. They further provide sensitivity analysis of the equilibrium strategies and system blocking probabilities to changes in the buffer size and the proportion of patient customers.

\section{References}

1. Economou, A.: The impact of information structure on strategic behavior in queueing systems. In: Anisimov, V., Limnios, N. (eds.) (2021) Queueing Theory 2, Advanced Trends. Wiley/ISTE, New York (2021)

2. Edelson, N.M., Hildebrand, K.: Congestion tolls for Poisson queueing processes. Econometrica 43, 81-92 (1975)

3. Ghosh, S., Hassin, R.: Inefficiency in stochastic queueing systems with strategic customers. Preprint (2021)

4. Hassin, R., Haviv, M.: To Queue or Not to Queue: Equilibrium Behavior in Queueing Systems. Kluwer Academic Publishers, Boston (2003)

5. Hassin, R.: Rational Queueing. CRC Press, Taylor and Francis Group, Boca Raton (2016)

6. Haviv, M., Oz, B.: Regulating an observable M/M/1 queue. Oper. Res. Lett. 44, 196-198 (2016)

7. Haviv, M., Oz, B.: Self-regulation of an unobservable queue. Manag. Sci. 64, 2380-2389 (2018)

8. Ibrahim, R.: Sharing delay information in service systems: a literature survey. Queueing Syst 89, 49-79 (2018)

9. Naor, P.: The regulation of queue size by levying tolls. Econometrica 37, 15-24 (2009)

10. Stidham, S., Jr.: Optimal Design of Queueing Systems. CRC Press, Taylor and Frances Group, Boca Raton (2009)

Publisher's Note Springer Nature remains neutral with regard to jurisdictional claims in published maps and institutional affiliations. 